\title{
Evaluation of Prevalence of Bacteria Helicobacter pylori in Potentially Malignant Disorders and Oral Squamous Cell Carcinoma
}

${ }^{1}$ Preethi Sharma, ${ }^{2}$ Madhuri Gawande, ${ }^{3}$ Minal Chaudhary

\begin{abstract}
Cancer is a complex disease that is variable in its presentation, development and outcome. The same heterogeneity and variability exist at the cellular and molecular level. Cancer is a multistep process during which cells undergo intense metabolic and behavioral changes, leading them to proliferate in an extreme and early way to escape supervision by the immune system and ultimately invades distant tissues to form metastases. G lobally, almost $20 \%$ of cancers are related to infectious agents. Several viruses with oncogenic potential stimulate cell proliferation leading to cancer in animals and humans. Viruses, in particular, have been found to play a major role in the process of cancer involving several organs. Bacterial species related with cancer etiology are varied; however, the infections they cause have common characteristics. The association between Helicobacter pylori and gastric carcinoma is well established but the association between $\mathrm{H}$. pylori and oral squamous cell carcinoma (OSCC) is not evident. Studies have also reported the existence of $\mathrm{H}$. pylori in the oral cavity, but whether the oral cavity serves as an extra gastric source for $\mathrm{H}$. pylori or carries the organism only transiently is debatable. Hence, with this aim in the mind, we conducted the present study to evaluate the incidence of $\mathrm{H}$. pylori in premalignant disorders and OSCC.
\end{abstract}

Keywords: Helicobacter pylori, Oral squamous cell carcinoma, Potentially malignant disorders.

How to cite this article: Sharma P, Gawande M, Chaudhary M. Evaluation of Prevalence of Bacteria Helicobacter pylori in Potentially Malignant Disorders and Oral Squamous Cell Carcinoma. World J Dent 2015;6(2):82-86.

\section{Source of support $\mathrm{Nil}$ \\ Conflict of interest: None}

\section{INTRODUCTION}

Oral cancers are related to the use of tobacco and alcohol consumption. Other factors include viral infections, infection with candida, etc. Bacterial species are associated

\footnotetext{
${ }^{1}$ Lecturer, ${ }^{2} \mathrm{P}$ rofessor and Head, ${ }^{3} \mathrm{P}$ rofessor and Dean

1,2Department of Oral Pathology, Sharad Pawar Dental College Wardha, Maharashtra, India

${ }^{3}$ Faculty of Dentistry, Sharad Pawar Dental College, Wardha Maharashtra, India
}

Corresponding Author: Preethi Sharma, Lecturer, Department of Oral Pathology, Sharad Pawar Dental College, Sawangi (M), Wardha, Maharashtra, India, Phone: 9764848712, e-mail: dr.preethisharma @ gmail.com with different cancers. Increasing evidence shows the association of bacteria with oral cancers. Helicobacter pylori is a microaerophilic Gram-negative spiral organism first isolated by Marshall and Warren from human gastric mucosa in 1983.

Helicobacter pylori bacteria can undergo coccoid transformation under hostile conditions and participate in the transmission of infection. ${ }^{2}$ Specific H. pylori characteristics, such as its gene diversity, helical morphology, citric acid cycle and lipopolysaccharide indicate the acclimination of the bacteria to the gastric mucosa. Newer methods, such as genomic comparision, analysis by transcriptomic method provides an accuracy on the molecular genetics of $H$. pylori. Infection can be diagnosed by invasive methods, e.g. culture, rapid urease test (RUT), ${ }^{2}$ histology and molecular methods or non-invasive, such as serology, urease breath test (UBT), stool antigen test and other methods.

The present study aims to assess prevalence of bacteria $H$. pylori in potentially malignant disorders and oral squamous cell carcinoma (OSCC).

\section{MATERIALS AND METHODS}

\section{Sample}

The study included 50 cases of normal individuals, 50 cases of histopathologically diagnosed cases of potentially malignant disorders and 50 cases of oral squamous cell carcinoma selected from the outpatient department (OPD) of the department of oral pathology.

The materials used for the study were saliva collector, petri plates, microaerophilic gas jar, campylobacter supplement, rapid urease broth, incubator, phosphate buffered saline. Unstimulated saliva was collected in a saliva collector after proper rinsing of the oral cavity. About $1 \mathrm{ml}$ of saliva was collected from every subject and control in a sterile saliva collector. Serial dilution of the specimen was carried out. The media was prepared as per the instructions given along with the media from the Himedia Laboratory Private Limited.

After preparation of the media, the diluted specimen was added on each plate containing media using streak culture method. All the plates are then placed in the 
microaerophilic gas jar. The gas jar is then placed inside the incubator at $37^{\circ} \mathrm{C}$ for 5 to 7 days. After 7 days, the colonies cultured on petri plates was subjected to rapid urease test (Fig. 1) for confirmation of bacteria. A number of colonies of bacteria grown on each plate were counted.

\section{RESULTS}

The microbial assessment of saliva in the respective groups was carried out by culturing of microorganism in the selective medium. The quantitative microbial assessment was done. The results were presented with detailed analysis of $H$. pylori counts in individual groups as descriptive statistics and their significant differences in the groups. We evaluated the presence of $H$. pylori in all the groups. The statistical tests used for the analysis of the result was the Chi-square test, Kruskal-Wallis test (Tables 1 and 2) (Graphs 1 and 2).

Table 1 depicts the correlation of number of colonies counted in leukoplakia and oral submucous fibrosis (OSMF). It was found that there was increased incidence of $H$. pylori count in OSMF when compared to leukoplakia.

Table 2 depicts the number of colonies counted in 50 cases of normal, premalignant conditions and OSCC. It was found that there was an increase in the number of

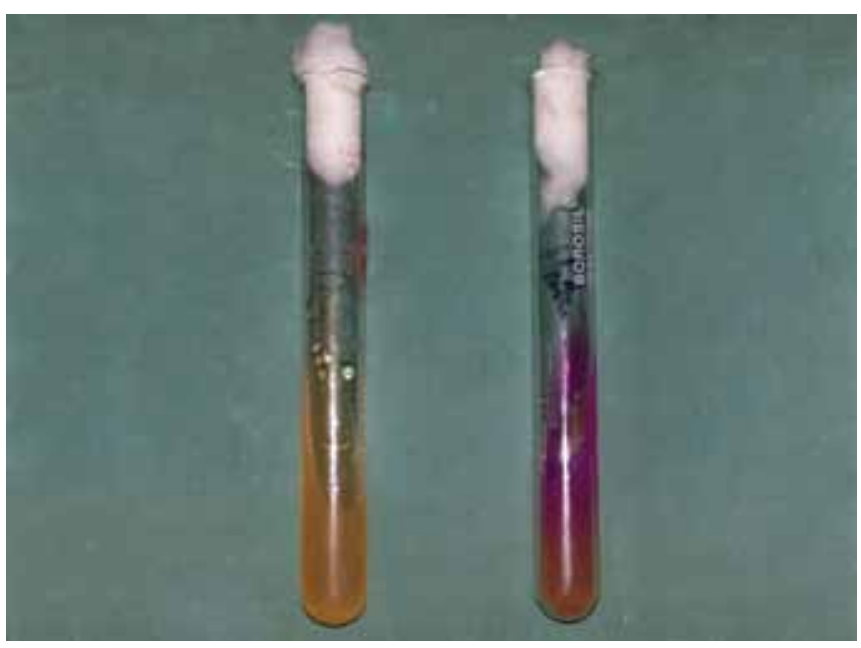

Fig. 1: Confirmatory biochemical rapid urease test

Table 1: Correlation of number of colonies in patients with leukoplakia and OSMF

\begin{tabular}{|c|c|c|c|c|c|c|}
\hline Diagnosis & $\begin{array}{l}\text { Number of } \\
\text { patients }\end{array}$ & 0 & $\begin{array}{l}1 \text { to } \\
2\end{array}$ & $\begin{array}{l}3 \text { to } \\
5\end{array}$ & $>5$ & $\begin{array}{l}\text { p-value } \\
\text { (with } \\
\text { normal) }\end{array}$ \\
\hline Leukoplakia & $29(58 \%)$ & 20 & 8 & 1 & 0 & $\begin{array}{l}7.52 \\
p=0.023,5\end{array}$ \\
\hline OSMF & $21(42 \%)$ & 8 & 13 & 0 & 0 & $\begin{array}{l}23.60 \\
p<0.0001,5\end{array}$ \\
\hline Total & $50(100 \%)$ & 28 & 21 & 1 & 0 & $\begin{array}{l}16.94 \\
p=0.0002, S\end{array}$ \\
\hline$\chi^{2}$-value & \multicolumn{6}{|l|}{6.21} \\
\hline p-value & \multicolumn{6}{|c|}{$0.044, S, p<0.05$} \\
\hline
\end{tabular}

H. pylori count in OSCC when compared to premalignant conditions and normal subjects.

\section{DISCUSSION}

In the present study, campylobacter supplement media (Skirrow's) was used to detect H. pylori. When the

Table 2: Correlation of number of colonies in normal, premalignant and OSCC patients

\begin{tabular}{llll}
\hline $\begin{array}{l}\text { Colonies } \\
\text { counted }\end{array}$ & $\begin{array}{l}\text { Normal } \\
\text { patients }\end{array}$ & $\begin{array}{l}\text { Premalignant } \\
\text { lesions }\end{array}$ & $\begin{array}{l}\text { Carcinoma } \\
\text { patients }\end{array}$ \\
\hline 0 & 46 & 28 & 18 \\
1 to 2 & 4 & 21 & 17 \\
3 to 5 & 0 & 1 & 14 \\
$>5$ & 0 & 0 & 1 \\
Total & 50 & 50 & 50 \\
$\chi^{2}$-value & - & 5.12 & 61.44 \\
p-value & - & $0.023, \mathrm{~S}$ & $\mathrm{p}<0.0001, \mathrm{~S}$ \\
& Premalignant & - & 50.82 \\
& lesions vs & & \\
& carcinoma & & \\
& patients & &
\end{tabular}

$\mathrm{p}<0.0001, \mathrm{~S}$

S: Significant

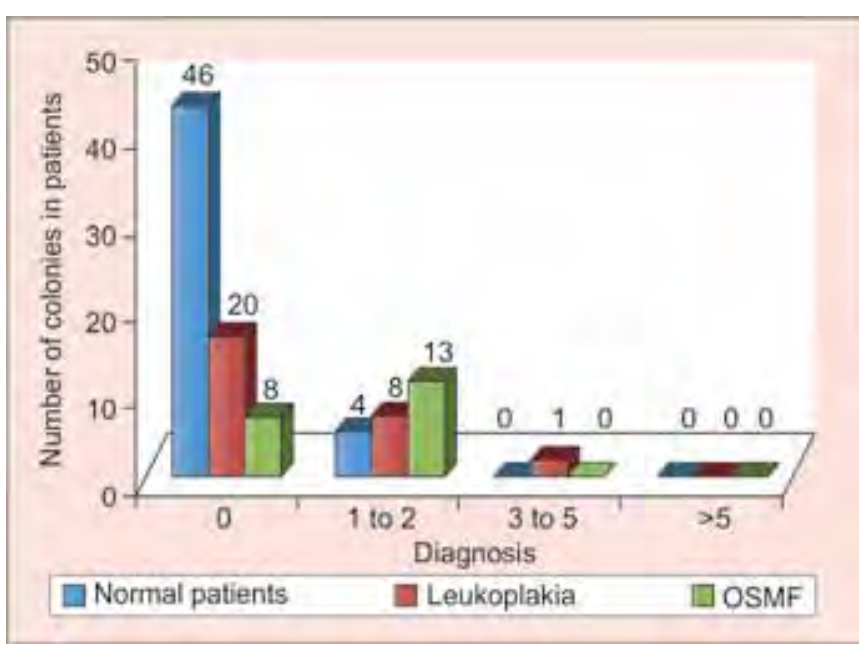

Graph 1: Correlation of number of colonies in patients with leukoplakia and OSMF

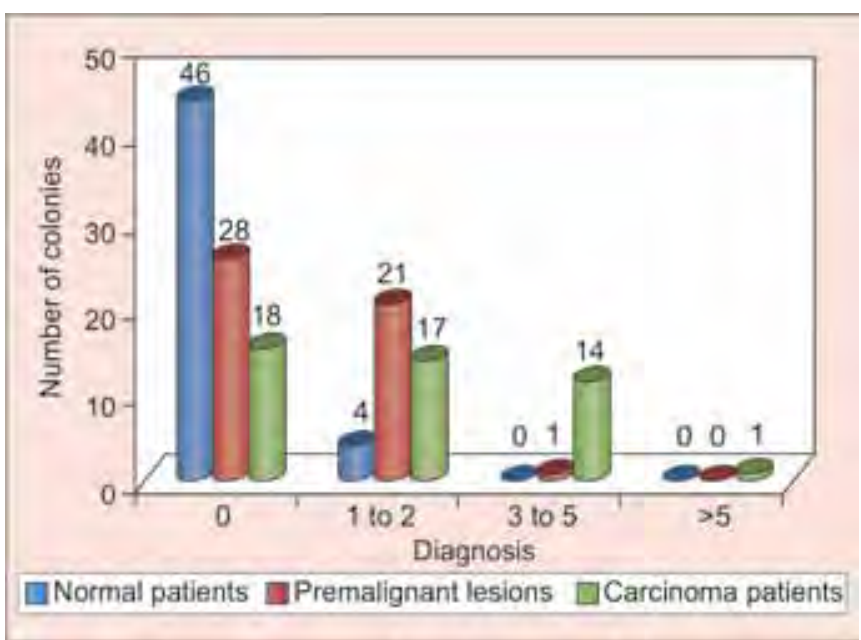

Graph 2: Correlation of number of colonies in normal, premalignant and OSCC patients 
prevalence of $H$. pylori count was compared between leukoplakia and OSMF, it was found that there was increased incidence of $H$. pylori count in OSMF compared to leukoplakia (Figs 2 and 3). The possible risk factors for increased prevalence of the count could be the habit of tobacco and tobacco with lime. The increase in number of colonies in OSMF probably could be attributed to other elements added to tobacco and lime in cases of patients addicted to commercially available tobacco pouches. The oral cavity harbors diversified microflora with more than 750 distinct bacterial taxa that colonise the host tissues. The presence of $H$. pylori in the oral cavity is transient. Bacterial biota becomes pathogenic when their balance is disturbed in the oral cavity. The growth of bacteria is influenced by a variety of factors, such as temperature, $\mathrm{pH}$, availability of nutrients and water, anatomy of oral structures, salivary flow and antimicrobial substances. ${ }^{3-5}$ The role of lime could be a source of this disturbance. Lime (calcium oxide in aqueous forms calcium hydroxide) alters the $\mathrm{pH}$ in the oral cavity probably over riding with

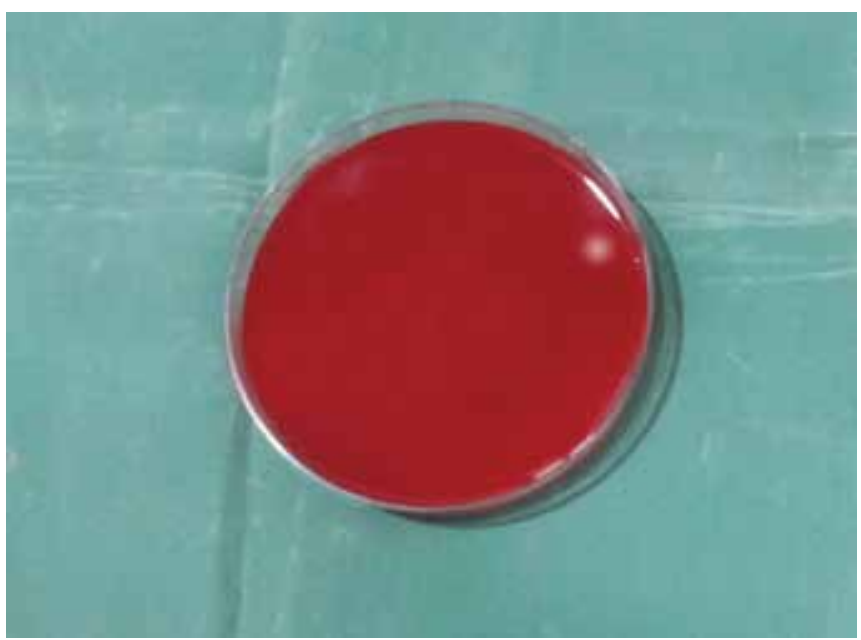

Fig. 2: Small, circular, convex, translucent colony of $\mathrm{H}$. pylori on Skirrow's media in leukoplakia

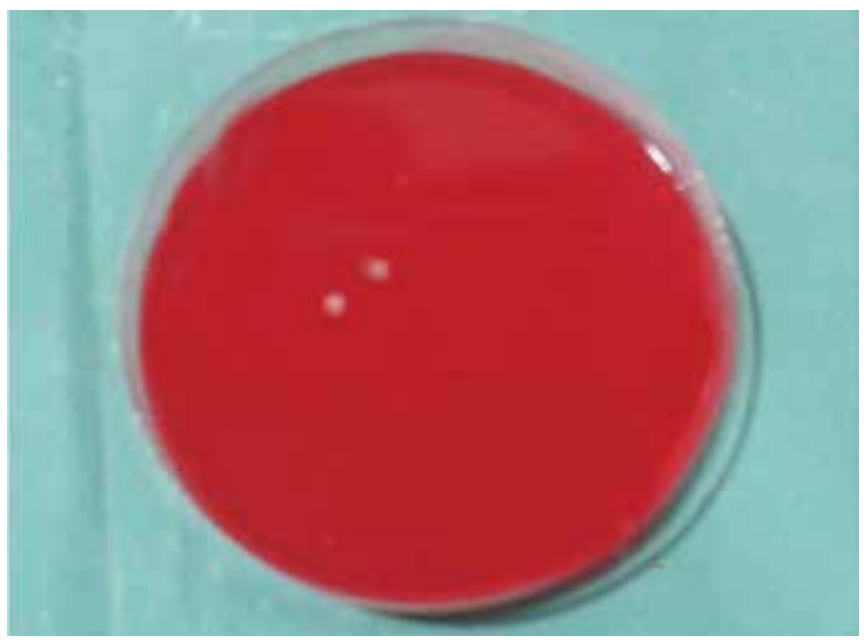

Fig. 3: Small, circular, convex, translucent colony of $\mathrm{H}$. pylori on Skirrow's media in OSMF the salivary buffering system and produces an alkaline environment which favors the growth of the bacteria. The presence of $\mathrm{Ca}(\mathrm{OH})_{2}$ in slaked lime also leads to the generation of reactive oxygen species which causes oxidative damage in the buccal mucosa cells of tobacco chewers. The oral cavity is a moist environment which is kept at a relatively constant temperature of 36 to $37^{\circ} \mathrm{C}$ and $\mathrm{pH}$ close to neutrality in most areas, and thus supports the growth of bacteria like H. pylori. ${ }^{4}$ Oral cancer associated with $H$. pylori infection evolves as a consequence of histological changes in the buccal mucosa due to chronic inflammation, which culminates initially into dysplasia and, at later stages, into cancer.

When the prevalence of $H$. pylori count was compared between normal, premalignant and OSCC patients, it was found that there was increased significance of H. pylori count in OSCC when compared to premalignant and normal patients (Fig. 4). This is in accordance with a study by Dayama et al (2011). ${ }^{6}$ The results of the study suggested a possible association of $H$. pylori with an increased risk of oral cancer. Tobacco could be the associated risk factor. The oral bacteria demonstrates specific tropism toward different biological surfaces in the oral cavity. This tropism suggests that different bacterial species have different receptors and adhesion molecules that dictates the colonisation of the different oral surfaces. Once the lesion is formed which may be spontaneous or due to underlying changes in the host tissues as a result of external factors, such as tobacco chewing and oral health, specific oral bacteria can colonise and induce inflammation.

The bacteria can lead to chronic infections or produce toxic metabolites that disturb the cell cycle leading to altered cell growth. Chronic infections induce cell transformation and DNA replication through the signaling pathways, such as mitogen-activated protein kinase (MAPK) pathway and cyclin D1, and increases

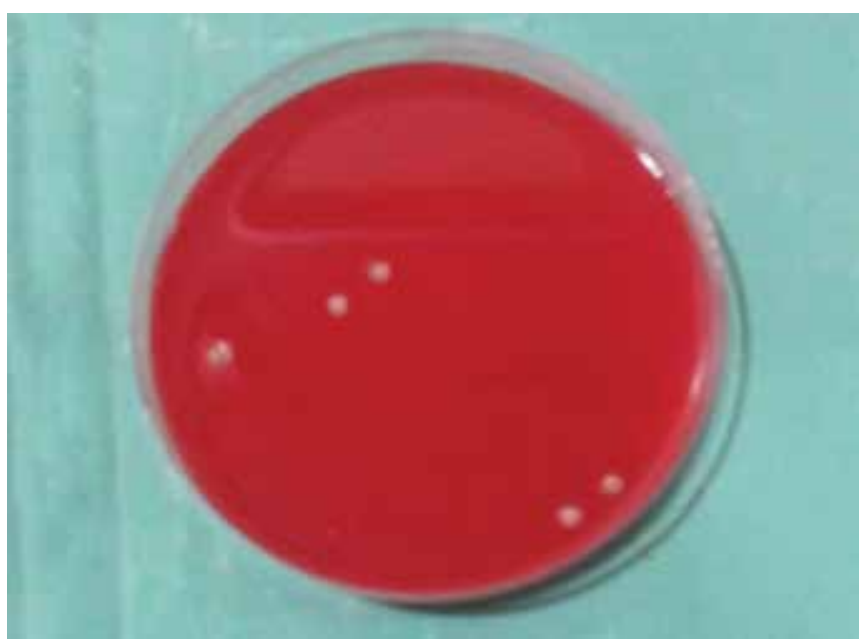

Fig. 4: Small, circular, convex, translucent colony of $\mathrm{H}$. pylori on Skirrow's media in OSCC 
the rate of cell proliferation and tumor development induced by genetic mutations. ${ }^{7}$ Several infections cause intracellular accumulation of the pathogen by modulating the expression of Bcl-2 family proteins leading to suppression of apoptosis or by inactivation of retinoblastoma protein, $\mathrm{pRb}$. This strategy provides an environment in which the intracellular pathogen can survive in spite of the defense by the host immune system to destroy the infected cells by apoptosis. Thus, it allows the partially transformed cells to evade the immune surveillance becoming tumorigenic. The pathogenicity and virulence of $H$. pylori is also determined by a set of genes (denoted as pathogenicity island or PIA), among which the presence of the cytotoxin associated gene cag $\mathrm{A}$ codes for protein that confers the pathogenic character to H. pylori strains. CagA+ H. pylori strains have strong association with cancer. ${ }^{8}$ The CagA protein of $H$. pylori has been shown to translocate into the cytoplasm of epithelial cells, where it mediates a number of cellular events including rearrangement of the cytoskeleton, induction of inflammatory mediators through the specific induction of proliferative and oncogenic proteins (via induction of oncogenic transcription factors like nuclear factor $\kappa B$ ), activating protein-1, phosphatidylinositol-3-kinase, signal transducer and activator of transcription (STAT)-3 that promote tumorigenic transformation. The prevalence of H. pylori infection in precancer and cancer lesions, along with its correlation with habits of tobacco and alcohol use can be considered as a potential risk factors. Thus, H. pylori directly and the immune/inflammatory response to $H$. pylori indirectly can influence the rate of epithelial cell proliferation, suggesting this bacterium may be an initiating step in oral carcinogenesis and an important co-carcinogenic factor. To summarise, $H$. pylori infection and chronic inflammation leads to the activation of multiple oncogenic pathways and malignant transformation. The role of inflammation, activation of protooncogenes, epigenetic mechanisms, local microenvironment and host genetic susceptibility together determine and promote the progression of cancer., ${ }^{9,10}$

Toxic metabolite products are produced in the H. pylori infected mucosa and this is another possible mechanism by which $H$. pylori induces oral carcinogenesis. ${ }^{11}$ Among the factors that influence epithelial changes in $H$. pylori infected patients are ROS and RNS. Excessive toxic metabolite production has been reported in human buccal mucosa infected with $H$. pylori, and correlates well with histological mucosal damage and with bacterial load. Helicobacter pylori itself has been reported to produce a large amount of superoxide anion $\left(\mathrm{O}_{2}^{*-}\right)$ in order to inhibit the bactericidal effects of nitric oxide (NO) synthesized by inflammatory cells. On the other hand,
$\mathrm{O}_{2}^{--}$might be passively produced by electrons leaking from the respiratory mitochondrial pathway of H. pylori, since $\mathrm{O}_{2}^{--}$production by $H$. pylori can be suppressed by cyanide $\left(\mathrm{CN}^{-}\right)$. The cytotoxicity of $\mathrm{O}_{2}^{--}$is moderate, but the cytotoxicity of hydroxyl radicals $(\mathrm{OH})$ produced via Fenton's reaction with metals and hydrogen peroxide $\left(\mathrm{H}_{2} \mathrm{O}_{2}\right)$ is much higher, and, therefore, $H$. pylori produced $\mathrm{O}_{2}^{*-}$ might indirectly induce epithelial cell injury leading to carcinogenesis. ${ }^{11}$

\section{SUMMARY}

Leukoplakia is 'a white patch or plaque that cannot be characterized clinically or pathologically as any other disease'. Oral submucous fibrosis is a chronic insidious disabling condition of the oral mucosa affecting any part of the mouth and rarely the pharynx, larynx and oesophagus. The malignant transformation rate of Leukoplakia is 0.13 to $17.5 \%$ and OSMF is $7.6 \%$. Head and neck squamous cell carcinoma are aggressive epithelial malignancies and are among the most commonest neoplasm in the world today. The tumor progression in epithelia has been classified as normal, hyperplastic (non- dysplastic), carcinoma in situ and invasive carcinoma.

There are very few scientific studies in the literature on the presence of bacteria $H$. pylori in the oral cavity in cohorts of patients with premalignant conditions and oral squamous cell carcinoma. Hence, we embark on this study to obtain baseline data on H. pylori carriage in oral cavity in premalignant conditions and OSCC. For this conventional diagnostic microbial procedures were used and the final identification of the bacteria was done by rapid urease test. The study comprised of 50 diagnosed cases of premalignant conditions, 50 diagnosed cases of OSCC and 50 cases of normal healthy individuals as control group.

The result of the present study can be summarized as follows:

- There was increase in H. pylori count in premalignant conditions as compared to normal subjects.

- The H. pylori count was increased in OSCC patients as compared to normal healthy individuals and patients with premalignant conditions.

\section{CONCLUSION}

The results of the present study confirm the phenomenon of high prevalence of oral colonization by $H$. pylori in premalignant conditions and OSCC patients.

Oral microbiological prevalence studies with frequently monitored, bigger population groups are required to clarify and confirm the present findings and its role in the etiology of OSCC. 


\section{REFERENCES}

1. Marshall BJ. Virulence and pathogenecity of H. pylori. J Gastroent Hepatol 1991;6:121-124.

2. Owen RJ. Helicobacter species classification and identification. British Medical Bulletin 1998;54(1):17-30.

3. Devi PU. Basics of carcinogenesis. Health Administrator 2010;18(1):16-24.

4. Marcotte H, Lavoie MC. Oral microbial ecology and the role of salivary immunoglobulin A. Microbiol Molecular Biology Review 1998;62(1):71-109.

5. Morii H, Nishizawa Y, Miki T. Effect of saliva on the growth of Helicobacter pylori. Osaka City Medical J 1999;45(1):15-23.

6. Dayama A, Srivastava V, Shukla M. Helicobacter pylori and oral cancer. Asian Pacific J Cancer Prevention 2011;12:1333-1336.
7. Rajeev, Chaudhary K, Pande S. Role of bacteria in cancer. South Asian J Cancer 2012;1(2):78-83.

8. Atherton JC. Helicobacter pylori virulence factors. British Medical Bulletin 1998;54(1):105-120.

9. Ding SZ, Goldberg JB. Helicobacter pylori infection, oncogenic pathways and epigenetic mechanisms in gastric carcinogenesis. Futur Oncology 2010;6(5):851-862.

10. PatilS, Rao RS, Sanketh DS, Sarode SC, Sarode GS. A universal diagnostic criteria for oral lichen planus: an exigency! Int J Contemp Dent Med Rev 2014. Article ID 041214, 2014. DOI: 10.15713/ins.ijcdmr.15

11. Handa O, Naito Y, Yoshikawa T. Redox biology and gastric carcinogenesis: the role of Helicobacter pylori. Redox biology and gastric carcinogenesis. Redox Report 2011;16(1):1-7. 